\title{
Standounkt
}

Wie können wir die Klimaziele im Verkehr erreichen?

\section{Die Verkehrswende braucht eine neue kommunale Planungskultur!}

\author{
Den Kommunen kommt in Deutschland eine wesentliche Auf- \\ gabe in der Verkehrswende zu. Verkehrsplanung ist die Grundlage \\ von Verkehrspolitik. Wie müssten sich ihre Prozesse verändern, \\ um die Verkehrswende effektiv zu unterstützen? Die „Guidelines \\ for Sustainable Urban Mobility Plans“ der EU geben darauf eine \\ systematische Antwort. \\ Von Siegfried Rupprecht
}

D ie „Fridays for Future“ und die andauernde Diskussion um städtische Luftqualität zeigen das wachsende öffentliche Bewusstsein um die Notwendigkeit der Verkehrswende. Auch in Deutschland sind die Kommunen einer der wesentlichen Akteure - aber können sie tatsächlich den erforderlichen Paradigmenwechsel in der Verkehrspolitik schaffen?

Die Rahmenbedingungen sind schwierig, denn Förderprogramme verfolgen oft technologische oder infrastrukturelle Förderschwerpunkte, Verfahrens- und Planungsvorgaben sind zahlreich, aber in der praktischen Entwicklung ihrer Verkehrspläne fühlen sich Kommunen häufig im Stich gelassen. Obwohl gerade Stadtverkehr mit anderen Politikfeldern eng vernetzt ist, fördern institutionelle Strukturen allzu oft das alte Ressortdenken. Skepsis ist also angebracht. Wie kann eine neue kommunale Verkehrsplanungskultur aussehen, die den Erfordernissen der Verkehrswende Rechnung trägt?

\section{Planung für das funktionale Stadtgebiet}

Ein wesentlicher Bestandteil des Verkehrs sind Pendlerströme, deren Quelle meist im Umland der Städte liegt. Erstellt eine Kommune also einen Mobilitätsplan ohne ihr Umland, erfasst sie nur einen Teil des Verkehrsgeschehens. Die Planung für das „funktionale“ Stadtge- biet stellt aufgrund der finanziellen, administrativen und politischen Probleme oft ein unüberwindbares Hemmnis dar. Ideal wäre eine enge Abstimmung der Flächennutzungsplanung auf der Ebene von Metropolregionen, die den beispielsweise nach dem Prinzip der „ÖPNV-orientierten Entwicklung " entstehenden Verkehr bereits an der Quelle reduzieren könnte.

\section{Kooperation mit anderen Politikbereichen}

Mangelhafte Kooperation der Planungsakteure führt auch in der Abstimmung von strategischen Zielen, Politiken und Finanzierungsinstrumenten zu einer wesentlichen Einschränkung der Effektivität. Verkehr hat einschneidende Auswirkungen auf alle Lebensbereiche, sodass Verkehrsplanung immer das Gesamtsystem Stadtregion auf Ziel- und Maßnahmenebene einbeziehen muss. Dies entspricht oft nicht mal ansatzweise der gängigen kommunalen Praxis.

Verkehrsplanung muss zukünftig viel aktiver Ziele in anderen Politikbereichen adressieren. Ein Mobilitätsplan muss neben verkehrlich und ökologisch wirksamen Maßnahmen auch soziale und wirtschaftliche Steuerungswirkungen entfalten - am besten durch integrierte Maßnahmenpakete, die eng über die kommunalen Politikfelder hinweg abgestimmt sind.

\section{Visionen für die Stadt von morgen}

Es ist zugegeben schwer, in Zeiten disruptiver Veränderungsprozesse Prognosen über effektive Lösungsstrategien abzugeben. Am Beispiel der mit vielen Unsicherheiten behafteten Diskussion um Szenarien für automatisierten Verkehr drängt sich die Frage auf, ob wir nicht intensiver über Grundfragen wie „In welcher Stadt sollen meine Kinder leben?" diskutieren sollten. Bricht man diese Frage auf klare Prioritäten und konkrete Zielkorridore herunter, kann Verkehrspolitik ihre normative Funktion viel effektiver erfüllen.

\section{Glaubwürdige Partizipation}

Dies kann aber nur effektiv gelingen, wenn die gesellschaftlichen Akteure einschließlich der Bürgerschaft aktiv und kontinuierlich in planerische Entscheidungsprozesse eingebunden sind. Partizipation ist viel mehr als ein abstraktes demokratisches Prinzip. Richtig angegangen, wird Bürgerbeteiligung im Planungsprozess zum Ideengeber, erhöht die Glaubwürdigkeit des Verfahrens und dient nicht zuletzt auch der Reduzierung politischer Risiken größerer Verkehrsprojekte durch Unterstützung selbst für zunächst unpopuläre Maßnahmen.

\section{Verkehrsplanung als Data Science}

Fakten sind die Grundlage guter Entscheidungen - auch wenn das aktuelle weltpolitische Geschehen dem zu widersprechen scheint. Fakten in der Verkehrsplanung beruhen auf Daten höchst unterschiedlicher Natur und Herkunft je besser ihre Qualität, desto bessere gesellschaftliche Wirkungen können auf Grundlage der darauf basierenden Entscheidungen entstehen. Vor der Nutzung solcher Datenquellen sind häufig erhebliche Hürden zu überwinden. Neben institutionellen Problemen fehlt es häufig an technologischen und rechtlichen Kompetenzen, der Umgang mit „Big Data“ ist dafür ein Beispiel von 
zunehmender Relevanz. Letztendlich muss sich die Verkehrsplanung stärker als Data Science begreifen, die von der ersten Problemanalyse über Szenarien, Maßnahmenmodellierung, Monitoring während der Umsetzung bis zu sozio-ökonomischen Wirkungsanalysen den Planungsprozess unterstützt.

\section{Nachhaltige Mobilitäts- planung in Deutschland}

Wir befinden uns in einem tief greifenden Transformationsprozess, der längst alle Lebensbereiche und Regionen der Welt erfasst hat - und zunehmende Mobilität ist Fluch und Segen zugleich. Mobilitätsplanung muss der Vielfältigkeit der Herausforderungen Rechnung tragen, die aus der großen Transformation resultieren. Unter dem Stichwort „Sustainable Urban Mobility Plan“ (SUMP) fördert die Europäische Union seit über zehn Jahren die Entwicklung von Modellprojekten und interkommunalen Lern- und Austauschprozessen. Seit 2013 gibt es eine formelle Empfehlung an die Mitgliedsstaaten und einen Leitfaden für Stadtverkehrsplanung. Diese „Guidelines for Sustainable Urban Mobility Plans“ wurden vor Kurzem auf der Grundlage eines umfassenden Konsultationsprozesses im Auftrag der Europäischen Union neu erstellt (Rupprecht Consult 2019).

Zahlreiche Städte in ganz Europa haben sich von den SUMP-Guidelines inspirieren lassen und auch in Lateinamerika und Asien findet das Konzept starkes Interesse. Die Grundlage von „Sustainable Urban Mobility Plans“ sind unter anderem die oben dargestellten Prinzipien: Planung für die „funktionale“ Stadt, enge Kooperation der Akteure mit dem Ziel integrierter Ziele, Politiken und Maßnahmenbündel, Entwicklung von Leitbildern und Zielvorgaben unter aktiver Beteiligung aller Akteure, faktenbasierte Planung und Evaluierung.

Auch in Deutschland arbeiten Verkehrsplanerinnen und Verkehrsplaner verstärkt auf der Grundlage des Konzepts des Sustainable Urban Mobility Plan oder verschmelzen es mit den Emp- fehlungen der deutschen Forschungsgesellschaft für Straßen- und Verkehrswesen zur Verkehrsentwicklungsplanung (Forschungsgesellschaft für Straßenund Verkehrswesen 2013). Diesen positiven Entwicklungen stehen allerdings Hemmnisse entgegen, die auf Landesoder Bundesebene angegangen werden müssen. Dazu zählen die Unterstützung der Kommunen in der Qualifizierung und im systematischen Austausch guter Praktiken, Förderung von integrierten, statt sektoralen Mobilitätskonzepten, klare Qualitätskriterien, obligatorische Wirkungsanalysen und eine Verpflichtung von Mobilitätsdienstleistern zum Datenaustausch.

Die Erfordernisse an eine Mobilitätsplanung, die die dringend erforderliche Neuorientierung des Stadtverkehrs effektiv unterstützt, sind hoch. Es bedarf grundlegender Veränderungsprozesse in den Stadtregionen. Die oftmals unzureichend ausgestatteten Akteure benötigen jede erdenkliche Unterstützung, um die Verkehrswende konsequent vorantreiben zu können.

\section{Literatur}

Forschungsgesellschaft für Straßen- und Verkehrswesen e. V. (Hrsg.) (2013): Hinweise zur Verkehrsentwicklungsplanung. Köln, FGSV Verlag.

Rupprecht Consult - Forschung \& Beratung $\mathrm{GmbH}$ (Hrsg.) (2019): Guidelines for Developing and Implementing a Sustainable Urban Mobility Plan, 2. Auflage. www.eltis.org/ mobility-plans/sumpguidelines

\section{AUTOR + KONTAKT}

Siegfried Rupprecht ist Geschäftsführer der Rupprecht Consult - Forschung \& Beratung $\mathrm{GmbH}$ und seit vielen Jahren im Bereich nachhaltiger Stadtverkehr tätig.

Rupprecht Consult - Forschung \& Beratung $\mathrm{GmbH}$, Clever Str. 13-15, 50668 Köln. Tel.: +49 22160605511 ,

E-Mail: s.rupprecht@rupprecht-consult.eu, Internet: www.rupprecht-consult.eu.

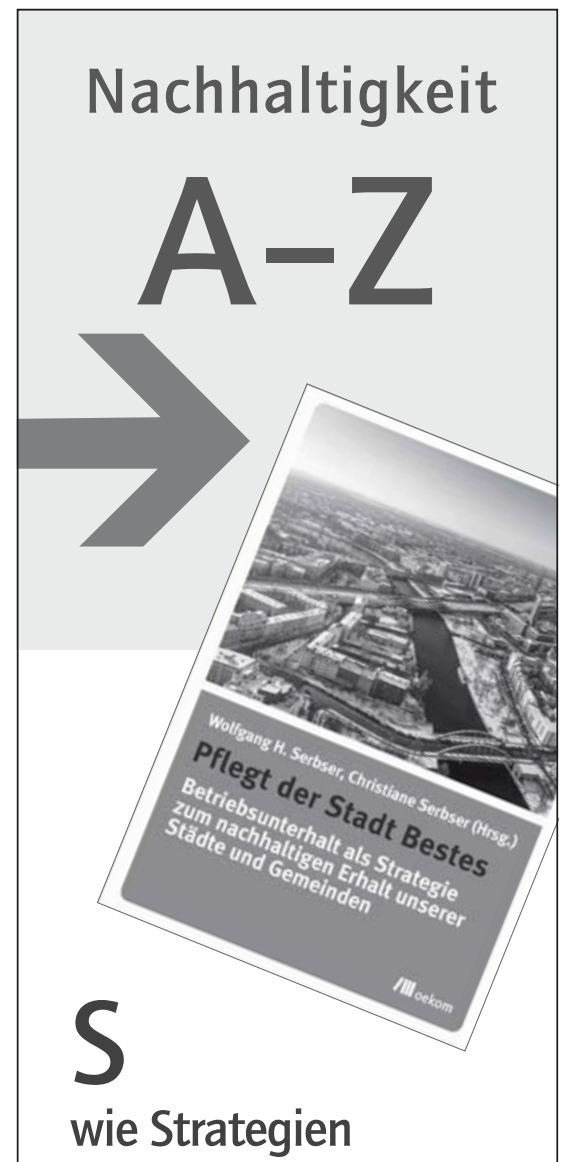

Was müssen Gebäude, Quartiere und Städte können, um zukunftsfest zu sein - um Schäden klein und Werte groß zu halten? Das ist nicht nur für die Immobilienwirtschaft wichtig, sondern auch für alle Organisationen, Unternehmen und Institutionen, die sich um Pflege und Erhalt von Bauten, Anlagen und Infrastrukturen kümmern. Hier werden sie fündig!

W. H. Serbser, C. Serbser (Hrsg.) Pflegt der Stadt Bestes Betriebsunterhalt als Strategie zum nachhaltigen Erhalt unserer Städte und Gemeinden 210 Seiten, broschiert, 22,- Euro, ISBN 978-3-96238-113-4

Erhältlich im Buchhandel oder versandkostenfrei innerhalb Deutschlands bestellbar unter www.oekom.de. Auch als E-Book erhältlich.

\section{/II oekom}

Die guten Seiten der Zukunft 